\title{
Environmental express monitoring of territory and water bodies at various stages of construction and improvement
}

\author{
Nikita Myazin ${ }^{1 *}$, Yuri Neronov ${ }^{2}$, Valentin Dudkin ${ }^{3}$, Vadim Davydov ${ }^{1,4}$, and Victoria \\ Yushkova $^{5}$ \\ ${ }^{1}$ Higher School of applied physics and space technologies, Peter the Great St.Petersburg Polytechnic \\ University, St.Petersburg, 195251, Russian Federation \\ ${ }^{2}$ D.I. Mendeleyev Institute for Metrology, St.Petersburg, 190005, Russian Federation \\ ${ }^{3}$ The Bonch-Bruevich Saint Petersburg State University of Telecommunications, St.Petersburg, \\ 193232, Russian Federation \\ ${ }^{4}$ All Russian Research Institute of Phytopathology, Moscow Region, 143050, Russian Federation \\ ${ }^{5}$ Saint Petersburg University of Management Technologies and Economics, St.Petersburg, 190103, \\ Russian Federation
}

\begin{abstract}
The article substantiates the need for continuous monitoring of the state of the environment both in the territories of the object under construction and in the areas of improvement works. The method of nuclearmagnetic spectroscopy is proposed, which allows carrying out ecological express monitoring of the state of condensed media (for example, water, silt, various mixtures, etc.) in real time. The results of ecological express monitoring are presented and recommendations for its more effective application are given.
\end{abstract}

\section{Introduction}

In conditions of high technological load on the environment, especially in large cities such as St. Petersburg, the role of enviromental monitoring of the territory and water bodies to ensure safe living increases [1-6]. One of the problems that arises in all countries of the world is the control of the state of the environment during the various works related to the construction and improvement [4, 6-10]. In most countries of the world (the Russian Federation is no exception), low-skilled workers, cheap and poorly packaged building materials are used to reduce the costs of work. Construction materials and industrial garbage are placed on the site with violations of their storage conditions. In the majority of situations this leads to a temporary littering of the site and penetration of hazardous substances into the soil, water drains, atmosphere and plants $[4,7,10-14]$. These substances begin to react with water (more often with precipitation) or with each other and pose a significant threat to living organisms $[3,10,11,12,15]$.

An important role in this situation is played by fast reliable methods of express control of condensed matter that can give information on the presence of deviations in a medium from

\footnotetext{
* Corresponding author: myazin.n@list.ru
} 
the standard state by in-situ measurements $[1,2,10,16-18]$. This makes it possible to quickly identify such foci of pollution.

Express control of the state of the environment during effective environmental monitoring should be implemented using portable devices. These instruments must measure the necessary parameters of the medium in a time not more than $10 \mathrm{~s}[11,16,17,20]$. The use of a mobile laboratory at these facilities is economically impractical, since measurements need to be carried out several times a day.

In addition, the mobile laboratory is not able to carry out most of the studies with the same accuracy that are provided in stationary laboratories. Most of the research in the mobile laboratory is carried out by chemical tests or chemical analysis, if appropriate reagents are available. With a complex combination of substances (for example, dissolved in water), it takes a lot of time and reagents to establish the composition of this mixture and it is not always possible to do it (errors often occur). The sample of the medium after such studies for subsequent work is unsuitable. And in a number of disputable situations, confirmation of the results of environmental monitoring in a stationary laboratory is required.

This led to the fact that strict requirements were imposed on the methods of express control: the study should not change the chemical composition and physical structure of the sample of the substance taken $[11,16,17]$. This is necessary to confirm the deviation detected in the study of a given sample of the substance, by high-resolution devices in stationary laboratories (for example, during customs control, product quality control or object contamination), as well as to establish the possible cause of this deviation. Mutual claims based on the results of express control can be very serious.

Nuclear magnetic spectroscopy relying on the use of nuclear magnetic resonance (NMR) allows one to solve the majority of the tasks associated with a rapid reliable express control of the state of condensed matter, including the tasks arising at environmental monitoring because it is highly precise and does not affect chemical properties and structure of the material being examined $[11,12,16,17,21-23]$.

\section{Specific features of environmental monitoring by the NMR technique}

Basic parameters of any condensed media by which a change in its aggregative state (a temperature increase, the presence of other substances, whether dissolved or not) can be instantly established are longitudinal $\mathrm{T}_{1}$ and transverse $\mathrm{T}_{2}$ relaxation times $[1,12,16,17,22$, 23]. Nuclear magnetic spectrometers allow one to perform non-contact measurements of relaxation times of a liquid medium, both in the stationary and flowing state [1, 17, 22-24]. The only condition for their application is the presence of a sufficient number of nuclei with magnetic moments in the medium under study, such as fluorine, lithium, sodium, carbon, boron, chlorine, phosphorus, etc. [17, 22-24]. Now there exists a very large number of condensed media containing protons or nuclei of other elements with large magnetic moments, which can be analyzed by NMR spectrometers [17, 21-24].

To avoid errors in determining the degree of deviation of the condensed medium state from the normal one, it is necessary to measure two relaxation constants $T_{1}$ and $T_{2}$ because mixtures of water, oil, alcohol-containing, and acidic products with hazardous substances frequently have $T_{1}$ equal to $T_{2}$ of the initial medium in some temperature interval. The knowledge of only $\mathrm{T}_{1}$ (without $\mathrm{T}_{2}$ ) for the mixtures also makes it impossible to determine the degree of deviation from the normal state of the medium because there are media and also mixtures in which different structural arrangements of one medium or mixture have different $\mathrm{T}_{1}[12,23]$.

The design and operation principle of small-sized NMR spectrometers and relaxometers have been considered in detail by the authors $[11,12,16,17]$ and other scientists [21-23]. By 
measuring the air temperature with a standard device with high accuracy, it is possible to compare the values of relaxation times $T_{1}$ and $T_{2}$ of the medium measured by the NMR spectrometer with the basic values to instantly draw a conclusion about its state $[11,12,16$, 17].

As an example of the device operation (express control of the ecological state of water), Figure 1 shows the NMR signals from tap water, to which ferric chloride $\mathrm{FeCl}_{3}$ was added, which is used in various construction works

(a)

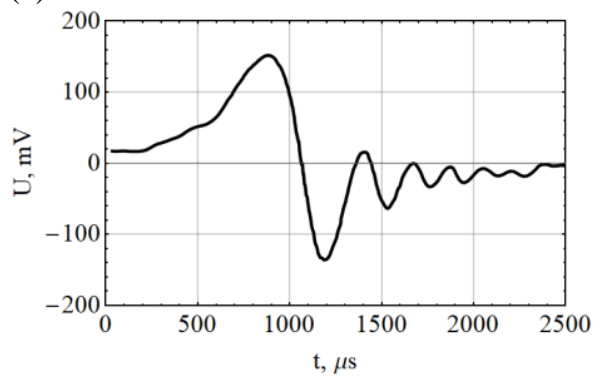

(c)

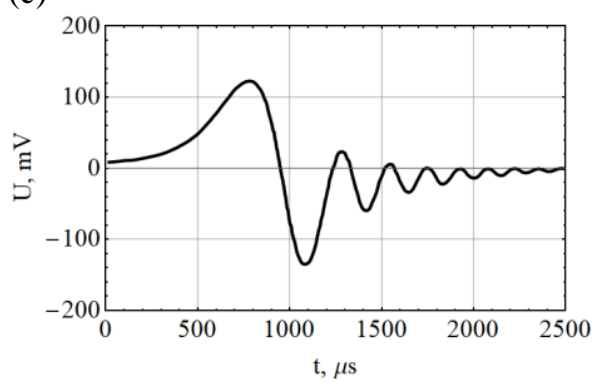

(b)

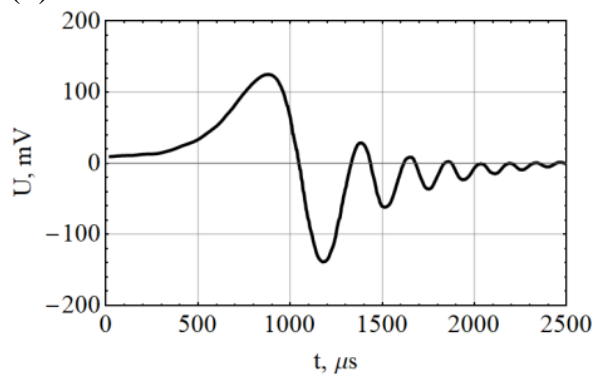

(d)

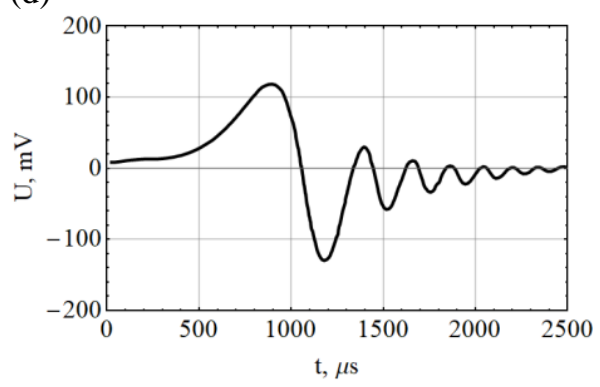

Fig. 1. NMR signals from tap water mixed in different proportions with iron chloride. Panels (a)-(d) correspond to $\mathrm{FeCl}_{3}$ concentrations of $0 \%, 1 \%, 4 \%, 10 \%$, respectively

Analysis of the NMR signals presented in Fig. 1, even without measuring the relaxation constants, provides information on the changes that occurred in water when iron chloride was added to it. However, in most cases the obtained visual results (Fig. 1) not always obvious. Therefore, it is necessary to measure the values of $\mathrm{T}_{1}$ and $\mathrm{T}_{2}$ for different environments and compare them with the standard.

\section{Results of environmental monitoring of the water body and their discussion}

The procedures for measuring $\mathrm{T}_{1}$ and $\mathrm{T}_{2}$ by the laboratory portable NMR spectrometer was tested at one of the water bodies situated in the $9^{\text {th }}$ January Memorial Park of St. Petersburg (Fig. 2). 

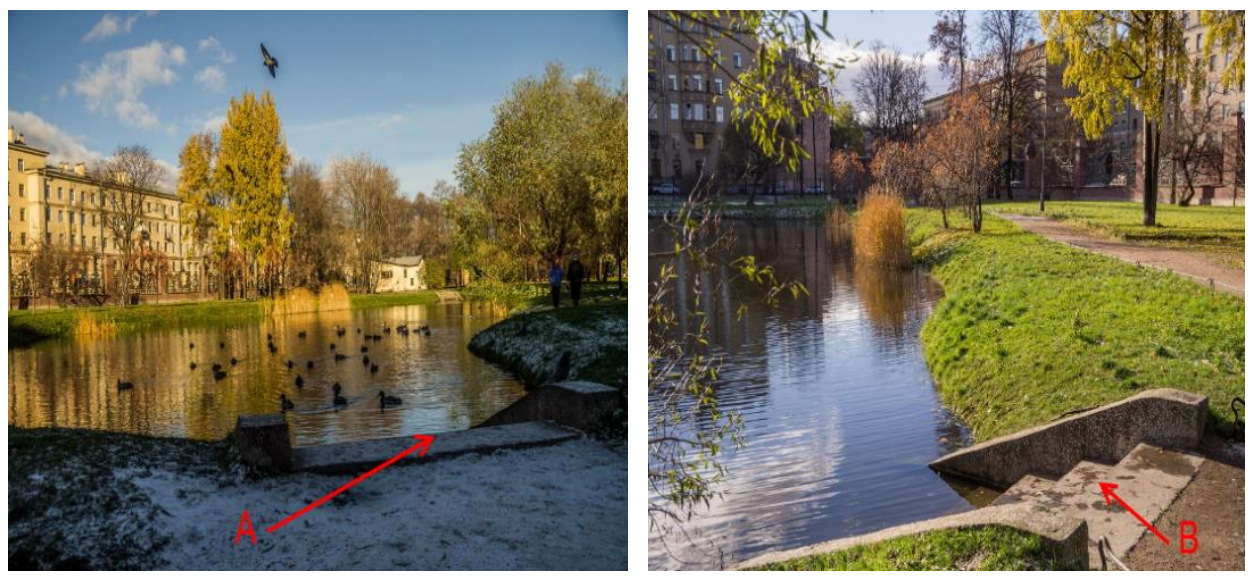

Fig. 2. The pond at which tests were performed

The water depth in this natural water formation with underwater springs is from 0.2 to $3.5 \mathrm{~m}$. Earlier crayfish could be met there, but now the pond is overgrown with weeds. During the tests a team of volunteers measured relaxation constants of water and bottom mud along the shore line at different distances $\mathrm{L}_{\mathrm{C}}$ from each other. The distance between the measuring points along the shore line was $0.3 \mathrm{~m}$. The coastline length $\mathrm{L}_{\mathrm{P}}$ was $116.2 \mathrm{~m}$ and $141.6 \mathrm{~m}$ if you moved around the pond to the right and left from the reference point A to point B (Fig. 2).

Figures 3, 4, 5 and 6 show dependences of relaxation constants of water and bottom mud at different points of the pond along its perimeter. The graphs show intervals $\Delta \mathrm{T}_{1}$ and $\Delta \mathrm{T}_{2}$, i.e., deviation of relaxation constants from those in the normal condition. These intervals are different for different liquid or viscous media and depend on temperature of the medium. The measurements were performed at the temperature of the medium $\mathrm{T}=274 \mathrm{~K}$. Since the volume of the medium used for measurements was not more than $1.5 \mathrm{ml}$, the sample temperature Ts became equal to the environmental temperature $\mathrm{T}$ in several seconds.

(a)

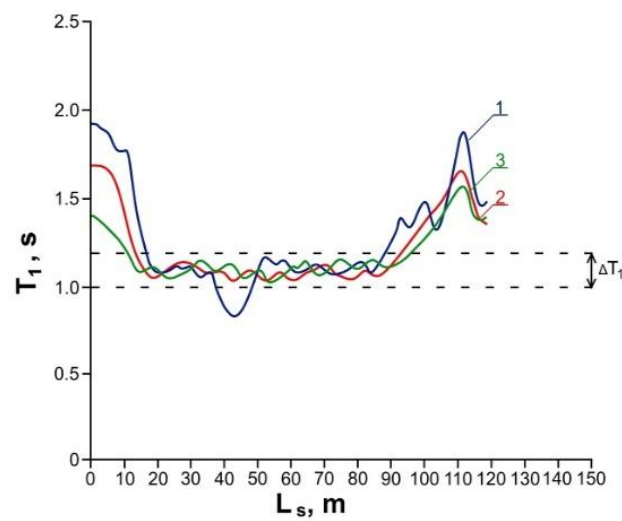

(b)

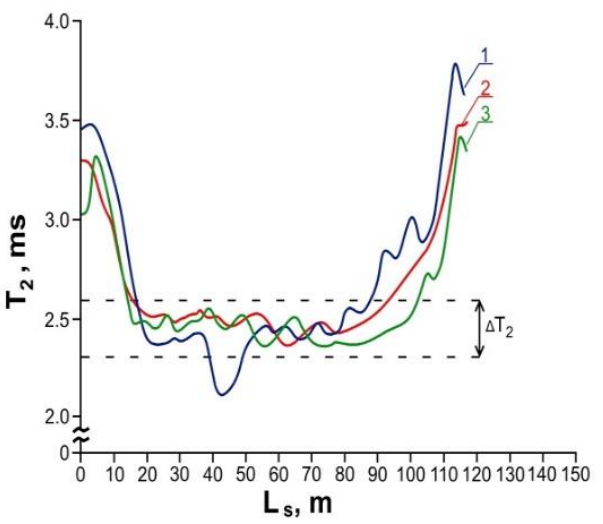

Fig. 3. Values of relaxation constants of water $\left((a)-T_{1} ;(b)-T_{2}\right)$ at different points of the pond along its perimeter (Fig. 2). The bypass of the pond was made from point A to point $\mathrm{B}$ along the right bank from A. Curves 1,2,3 correspond to $\mathrm{LC}_{\mathrm{C}}=5,15$, and $30 \mathrm{~cm}$, respectively 
(a)

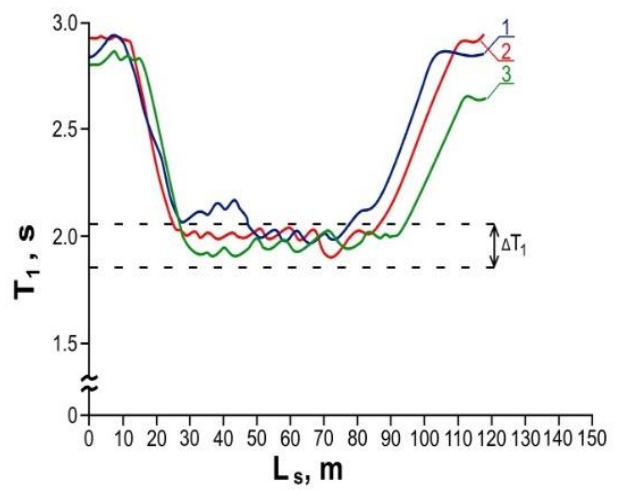

(b)

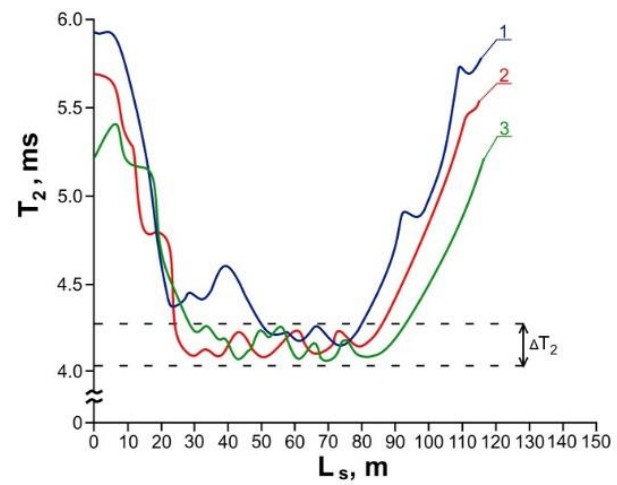

Fig. 4. Values of relaxation constants of bottom mud ((a) $\left.-T_{1} ;(b)-T_{2}\right)$ at different points of the pond along its perimeter (Fig. 2). The bypass of the pond was made from point $\mathrm{A}$ to point $\mathrm{B}$ along the right bank from A. Curves 1,2,3 correspond to $\mathrm{L}_{\mathrm{C}}=5,15$, and $30 \mathrm{~cm}$, respectively

(a)

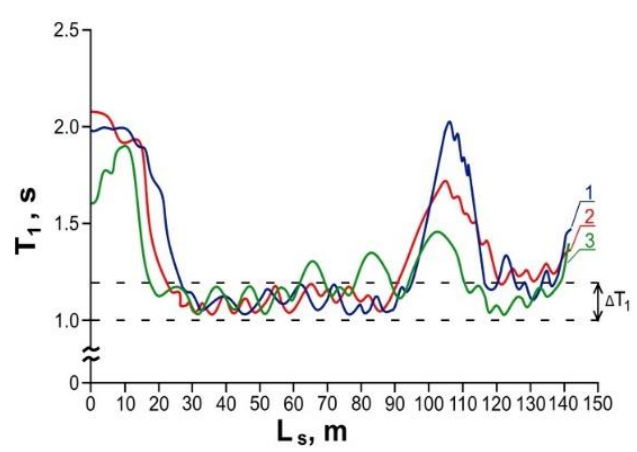

(b)

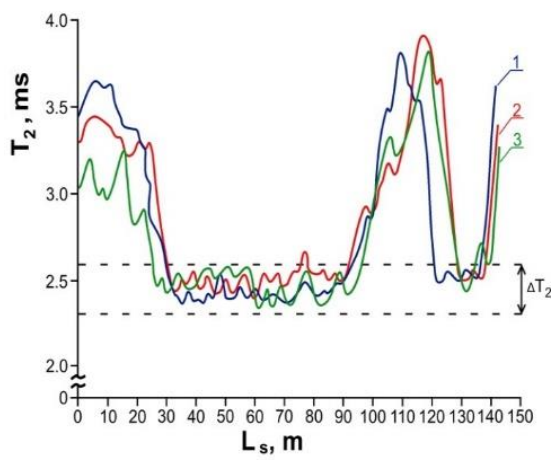

Fig. 5. Values of relaxation constants of water $\left((a)-T_{1} ;(b)-T_{2}\right)$ at different points of the pond along its perimeter (Fig. 2). The bypass of the pond was made from point A to point B along the left bank from A. Curves 1,2,3 correspond to $\mathrm{LC}_{\mathrm{C}}=5,15$, and $30 \mathrm{~cm}$, respectively

(a)

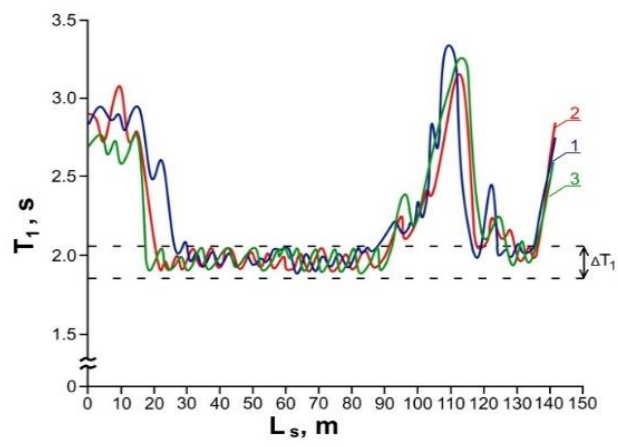

(b)

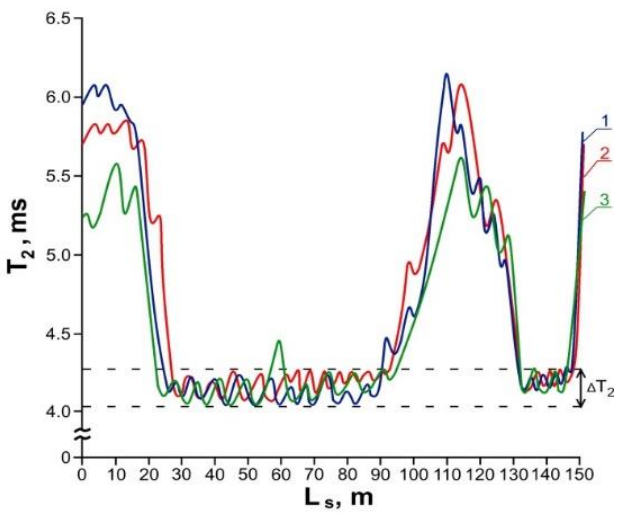

Fig. 6. Values of relaxation constants of bottom mud ((a) $\left.-T_{1} ;(b)-T_{2}\right)$ at different points of the pond along its perimeter (Fig. 2). The bypass of the pond was made from point $\mathrm{A}$ to point $\mathrm{B}$ along the left bank from A. Curves 1,2,3 correspond to $\mathrm{LC}_{\mathrm{C}}=5,15$, and $30 \mathrm{~cm}$, respectively 
A large number of deviations of relaxation constants from those of water and bottom mud in the normal condition were revealed though the water was transparent (Fig. 2). Experimental data showed that in the case of an acute pollution two relaxation constants deviated from the "normal" ones. Polluted areas can be found from the dependences shown in Fig. 3, 4, 5 and 6. Additional analysis of the samples is required for the cases of small deviations. Causes of pollution should be established. In some cases, contaminants can be localized and neutralized with time in a natural way. Then no territory cleaning-up is required.

In one of the pond areas, $\mathrm{T}_{1}$ was found to be twice as high as the "normal" value, while $\mathrm{T}_{2}$ did not significantly increase. The bottom mud samples were characterized by appreciably increased $T_{1}$ and $T_{2}$ relaxation constants. The water and bottom mud samples with deviations of relaxation constants were investigated at a stationary X-ray S2 RANGER spectrometer (BRUKER) at a laboratory of St. Petersburg National Mineral Resources University (University of Mines). It was found that the water and bottom mud contained a dissolved substance with the composition similar to that of citric acid. The samples from other pond areas were found to contain different concentrations of building lime, gasoline of all grades, varnishes, technical oils and fats, zinc, iron, manganese, and so on. In two samples mercury was detected.

Having completed the research activities, the volunteers cleared the pond and thoroughly inspected the extracted bottom mud, algae, etc. For comparison, the pond areas for which no deviation of relaxation constants was detected were examined. Waste of different kinds was found in the bottom mud (for example, bottles, building stone, etc.). This waste does not affect relaxation constants of the medium where it is found, though it can be harmful.

Further investigation and statistical processing of new results and also their comparison with the results of the environmental monitoring we obtained after cleaning the pond can be helpful in establishing other causes of the pond pollution in addition to the works connected with landscaping and building.

\section{Conclusions}

The experimental results have shown that NMR spectroscopy is an appropriate and effective technique for environmental express monitoring of building sites.

It should be noted that the ecological monitoring of the pond including a full analysis of the samples by an X-ray spectrometer would need 5156 containers for the samples. 28 samples can be loaded simultaneously into a S2 RANGER spectrometer. The total time for analysis of the sample (including sample reloading) is 15-20 minutes. So the analysis would require not less than 47 hours of continuous operation. The use of the express analysis method allowed us to conduct the necessary research by the S2 RANGER during 5 hours. In some areas of the pond where a danger of pollution was detected, the density of sampling was twice as high.

This indicates that the express control of building sites by portable NMR spectrometers is highly cost-effective. The operational efficiency of environmental monitoring of objects including identification of pollution foci by this method is also high.

\section{References}

1. V. Davydov and N. Myazin, Meas. Tech+. 60(2) 183-189 (2017)

2. N. Myazin, S. Logunov, V. Davydov, V. Rud', N. Grebenikova and V. Yushkova, J. of Phys.: Conf. Series, 929(1) 012064 (2017)

3. V. Arkhipov, Instrum. Exp. Tech+. 55 692-695 (2012). 
4. T. Polivanova, S. Polivanova, N. Kobelev, A. Akul'shin and V. Kobelev, Appl. Mech. Mater., 725-726 1332-1337 (2015)

5. N. Grebenikova, K. Smirnov, V. Artemiev, V. Davydov and S. Kruzhalov, J.of Phys.: Conf. Series, 1038(1) 012089 (2018)

6. R. Davydov, V. Antonov, D. Molodtsov and A. Trebukin, Adv. Intell. Syst., 692 915-920 (2018)

7. D. Nemova, R. Bagautdinov and A. Mushnikov, Procedia Engineer., 117(1) 1120-1126 (2015)

8. V. Mushchanov, V. Sievka, A. Veshnevska and D. Nemova, Procedia Engineer., 117(1) 1018-1026 (2015)

9. V. Maslak, N. Nasonkina, V. Sakhnoskaya, S. Antonenko and D. Nemova, Procedia Engineer., 117(1) 985-994 (2015)

10. S. Bezborodov and N. Zemlynaya, Appl. Mech. Mater., 725-726 1350-1356 (2015)

11. V. Davydov, V. Dudkin, N. Myazin and V. Rud', Instrum. Exp. Tech+, 61(1) 140-147 (2018)

12. V. Davydov, N. Myazin and T. Davydova, Russian J. of Nondestructive Testing, 53(7) 520-529 (2017)

13. M. Petrichenko, N. Vatin, D. Nemova, N. Kharkov and A. Staritcyna, Appl. Mech. Mater., 627 297-303 (2014)

14. N. Vatin, M. Petrichenko, D. Nemova, A. Staritcyna and D. Tarasova, Appl. Mech. Mater., 633-634 1023-1028 (2014)

15. E. Nepomnyashchaya, E. Savchenko, E. Velichko and E. Bogomaz, J. of Phys.: Conf. Series, 956(1) 012009 (2017)

16. N. Myazin, V. Davydov, V. Yushkova, T. Davydova and V. Rud', J. of Phys.: Conf. Series, 917(4) 042017 (2017)

17. V. Davydov, V. Dudkin, and A. Karseev, J. Appl. Spectrosc., 82(5) 794-800 (2015)

18. M. Baranov, E. Velichko and E. Aksenov, J. of Phys.: Conf. Series, 917(6) 062059 (2017)

19. E. Simonenko, V. Starkov, L. Molodkina and A. Chusov. Appl. Mech. Mater., 725-726 1357-1362 (2015)

20. E. Nepomnyashchaya, A. Cheremiskina, E. Velichko, E. Aksenov and E. Bogomaz, J. of Phys.: Conf. Series, 643(1) 012018 (2015)

21. J. Vervoort, J. van Leerdam, G. Stroomberg and P. de Voogt, Environ. Sci. Technol., 48 (21) 12791-12799 (2014)

22. S. D'yachenko, I. Kondrashkova and A. Zhernovoi, Tech. Phys+. 62(10) 1602-1604 (2017)

23. S. Dyachenko, M. Vaseshenkova, K. Martison, I. Cherepkova and A. Zhernovoi, Russ. J. Appl. Chem+., 89(5) 690-696 (2016)

24. V. Davydov, V. Dudkin and N. Myazin, J. Commun. Technol. El+., 61(10) 1159-1165 (2016) 